\title{
Understanding and predicting viscous, elastic, plastic flows
}

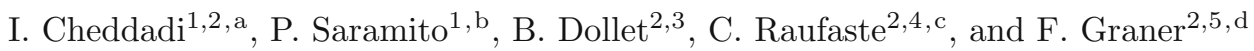

1 Laboratoire Jean Kuntzmann, UMR 5524 Université J. Fourier - Grenoble I and CNRS, BP 53, F-38041 Grenoble cedex 09, France

${ }^{2}$ Laboratoire de Spectrométrie Physique, UMR 5588 Université J. Fourier - Grenoble I and CNRS, BP 87, F-38402 Martin d'Hères cedex, France

3 Institut de Physique de Rennes, UMR 6251 Université Rennes 1 and CNRS, Campus Beaulieu, Bâtiment 11A, F-35042 Rennes cedex, France

4 Physics of Geological Processes, University of Oslo, Sem Selands vei 24, NO-0316 Oslo, Norway

5 BDD, Institut Curie, CNRS UMR 3215 and INSERM U 934, 26 rue d'Ulm, F-75248 Paris cedex 05, France

Received 11 July 2010

Published online: 7 January 2011

(c) The authors 2011. This article is published with open access at Springerlink.com

\begin{abstract}
Foams, gels, emulsions, polymer solutions, pastes and even cell assemblies display both liquid and solid mechanical properties. On a local scale, such "soft glassy" systems are disordered assemblies of deformable rearranging units, the complexity of which gives rise to their striking flow behaviour. On a global scale, experiments show that their mechanical behaviour depends on the orientation of their elastic deformation with respect to the flow direction, thus requiring a description by tensorial equations for continuous materials. However, due to their strong non-linearities, the numerous candidate models have not yet been solved in a general multi-dimensional geometry to provide stringent tests of their validity. We compute the first solutions of a continuous model for a discriminant benchmark, namely the flow around an obstacle. We compare it with experiments of a foam flow and find an excellent agreement with the spatial distribution of all important features: we accurately predict the experimental fields of velocity, elastic deformation, and plastic deformation rate in terms of magnitude, direction, and anisotropy. We analyse the role of each parameter, and demonstrate that the yield strain is the main dimensionless parameter required to characterize the materials. We evidence the dominant effect of elasticity, which explains why the stress does not depend simply on the shear rate. Our results demonstrate that the behaviour of soft glassy materials cannot be reduced to an intermediate between that of a solid and that of a liquid: the viscous, the elastic and the plastic contributions to the flow, as well as their couplings, must be treated simultaneously. Our approach opens the way to the realistic multi-dimensional prediction of complex flows encountered in geophysical, industrial and biological applications, and to the understanding of the link between structure and rheology of soft glassy systems.
\end{abstract}

\section{Introduction}

Materials such as pastes [1] or polymer solutions display both solid-like and liquid-like behaviors [2]; they are successfully described by visco-elastic (VE) or visco-plastic (VP) models. However, we still lack testable predictions of the time- and space-dependent flows of soft glassy materials [3-6], that are made of disordered assemblies of deformable, rearranging units $[3,7,8]$. It had been suggested

\footnotetext{
a Present address: INRIA Paris - Rocquencourt, Domaine de Voluceau, Rocquencourt, B.P. 105, 78153 Le Chesnay, France.

b e-mail: pierre.saramito@imag.fr

c Present address: Laboratoire de Physique de la Matière Condensée, UMR 6622 CNRS and Université Nice-Sophia Antipolis, Parc Valrose, F-06108 Nice Cedex 2, France.

d e-mail: francois.graner@curie.fr
}

that fluctuations remain relevant even at large scale, in which case detailed statistical theories of long-range correlations and avalanches would be required $[9,10]$. This view is challenged by recent experiments which suggest that even these materials can be treated as continuous materials described by tensorial equations [3,11]; thus in principle partial differential equations could lead to the long-awaited predictions.

Based on our experience with foams, we believe that the reason of the difficulty comes from the fact that these materials are simultaneously viscous, elastic, and plastic (VEP). Under small deformation, a foam reversibly comes back to its shape; at large deformation, it can be irreversibly sculpted and gets a new shape; under an increasing deformation rate, it irreversibly flows, with an increasing viscous stress [12-16]. Existing continuous models of 
foam flows include either a phenomenological scalar description $[17,18]$, or a complete tensorial description of the elasticity [19] or plasticity [7]. Overall, continuous VEP models tend to successfully reproduce some experimental measurements, such as elastic and loss moduli, or compliance (see for instance refs. $[20,19,21,15,8]$ ). In the case of other complex fluids, for instance dense colloidal suspensions [22,23] or wormlike micelles solutions [24], tensorial VEP models have also been developed, but tests were restricted to simple geometries: steady shear, uniaxial elongation, cylindrical Couette flow.

We want here to understand and predict VEP flows, assuming that material properties are known. We thus work downhill of statistical models which predict the material properties from the microstructure [25]. Our approach is to test whether a continuous VEP model can capture the essence and complexity of the flow properties, especially the elasticity and its advection by the flow.

We thus need a VEP model as simple as possible: with linear and isotropic coefficients, without fluctuations nor long-range interactions. We require to use only physically relevant parameters, which in principle are measurable. We need a tensorial model in a multidimensional space (what follows applies both in 2D and 3D). We need a closed system of equations: constitutive equations, specific to the material under consideration; generic conservation laws, in the spirit of hydrodynamics; and closure equations, to unify the solid and liquid descriptions.

To really test a model, and also lead to practical applications, we address the full spatial and orientational heterogeneity of a flow. We need to investigate a controlled, reproducible flow which displays significantly different $\mathrm{V}, \mathrm{E}$ and $\mathrm{P}$ contributions. It should involve a large range of shear rates, of tensorial orientations, and of elastic deformations. It should display elastic deformations either parallel, perpendicular or at odd angles with respect to the shear rate. Most discriminant, it should actually depend on two or more dimensions of space, so that the advection of elastic stress couples the shear and normal stress components (see eqs. (8), (9)). This latter point, overlooked by most models, is crucial to test whether the elastic stress could be entirely determined by the shear rate, leading to a VP rheology (such as Bingham [26] or HerschelBulkley [27]). Recent experiments [28-30] suggest that this is not the case: in fact, the stress, the shear rate and the elastic deformation should be treated as independent variables, so that a full VEP treatment is required.

One such flow is the well-documented flow around an obstacle [31,32] (fig. 1). It displays a strong spatial heterogeneity, simultaneous VEP behaviors, a large range of elastic deformations, several elongation and rotation rates, and various relative orientations of the relevant tensors [7]. It enables to follow a bubble at different stages, while it stretches, then while it relaxes: thus, even in a steady flow, transient effects and relaxation times are apparent. It is classically used as a stringent test to discriminate between different models [33] (fig. 2).

\section{(a)}

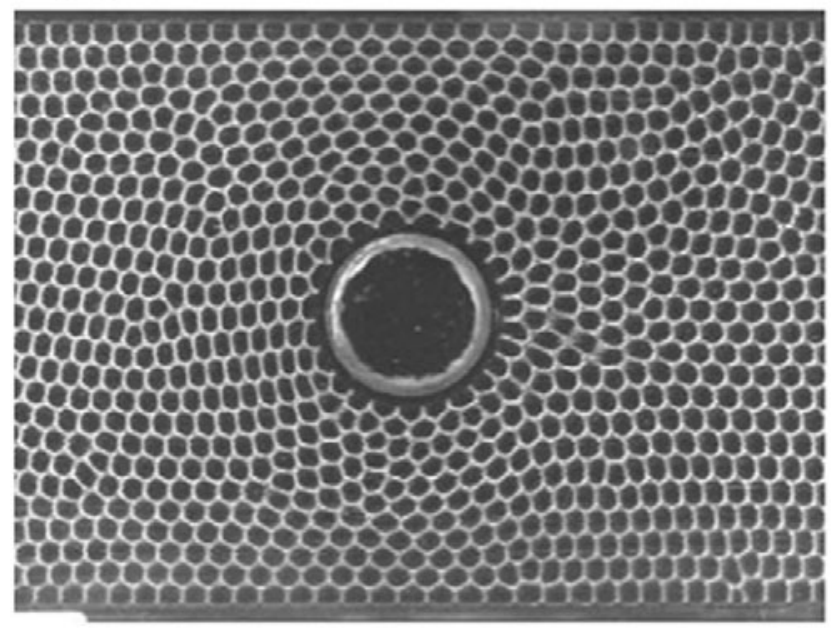

(b)

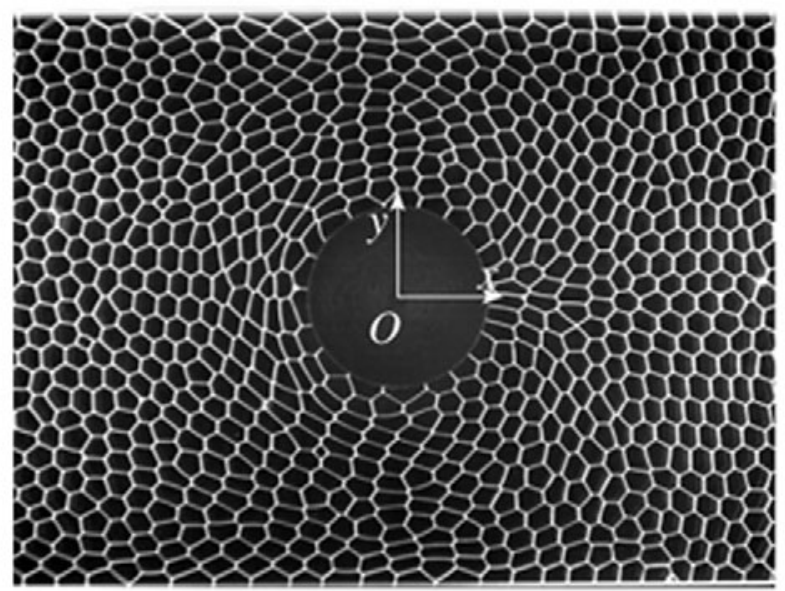

Fig. 1. Top view of the experiments. The foam is quasibidimensional, the flow (from left to right) is exactly bidimensional. The channel is rectangular: length $1 \mathrm{~m}$ (only partially shown), width $10 \mathrm{~cm}$. The obstacle is circular, with radius $R=1.5 \mathrm{~cm}$. (a) Wet foam [11], liquid fraction $\phi=7 \%$, entrance velocity $V=1 \mathrm{~cm} / \mathrm{s}$. (b) Dry foam, $\phi=1.2 \%, V=0.6 \mathrm{~cm} / \mathrm{s}$. The definition of axes $x$ and $y$ is superimposed on the obstacle. The origin $O$ is in the middle of the obstacle.

We use foams as model systems of VEP materials. Experiments with foams or emulsions, especially in 2 dimensions, enable an easy, simultaneous visualisation of the micro-structure (bubbles or droplets, which act as tracers of velocity and deformation) and the large scale (global flow heterogeneities).

To compare mesurements from discrete experiments with continuous predictions from partial differential equations, we use the experimental tools we have developed [7]. $\mathrm{V}, \mathrm{E}$ and $\mathrm{P}$ contributions are expressed in the same units, favoring a unified description of solid and liquid behaviors; each of them is valid in all regimes (so that, e.g. 


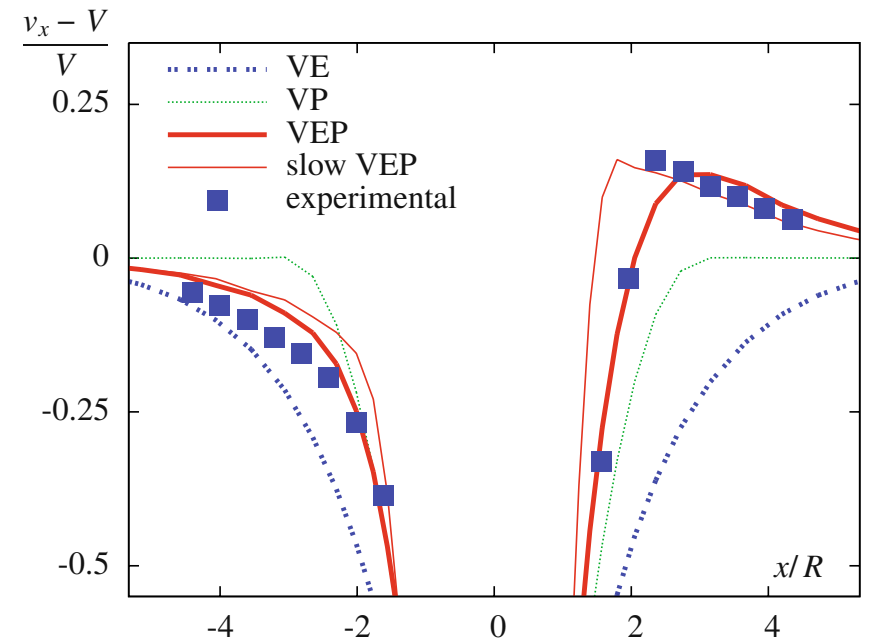

Fig. 2. Comparison of the flow around a circular obstacle with different models. Reduced velocity $\left(v_{x}-V\right) / V$ along axis $y=0$ in wet foam experiment (closed squares, measured in fig. 1a) is compared with the VEP calculation (present model, $\varepsilon_{Y}=0.1, \lambda=0.2 \mathrm{~s}, \eta_{1} / \eta_{2}=0.1, k=0$ ), slow VEP (entrance velocity $V$ divided by 20), VP (Bingham model [26], with the same Bingham number), VE (Oldroyd model [34], with the same $\lambda$ ).

elastic contributions can be measured even out of the elastic regime). These are local (in situ) measurements which link the foam structure and rheology.

Our plan is as follows. In order to make this paper self-contained, sect. 2 recalls Saramito model [21]; here we write it by emphasizing how it unifies solid and liquidlike behaviours. Section 3 briefly recalls the experimental methods. It explains the careful resolution algorithm we had to develop to deal with the strong non-linearities of VEP equations, and a well-controlled space and time discretisation. Section 4 first fits the solutions to a wet foam flow, fixing the values of the parameters. It then turns to predictions of a foam with a higher yield strain, which accurately match a dry foam flow. Section 5 includes concluding remarks.

\section{Model}

\subsection{Characteristics and past results of the model}

We choose to use Saramito's VEP model [21] because it has a positive dissipation, thanks to the convexity of its energy function. This implies that, at least for small deformations, it obeys by construction the second principle of thermodynamics. It includes as limiting cases both the VP Bingham model [26] and the VE Oldroyd model [34], and generalises them to VEP. It can extend to large deformations and high velocities.

It has already been used for space- and time-dependent predictions [21]. It has first been used to solve simple cases, such as steady uniaxial elongation. It has also been applied to oscillatory regimes, and calculations of $G^{\prime}, G^{\prime \prime}$, rigidity and loss moduli.

It has then been implemented to calculate both timedependent and steady shear Couette flows, which depend on one space variable (circular [35] or planar [36] geometries). It involves two strong non-linearities, intrinsic to VEP flows, and thus independent of the model: one because the plasticity appears above a yield point (eq. (3)), and the other because of the advection of elastic stress (eqs. (8), (9)). Despite these unavoidable difficulties, the model has been solved [37-39]. The resulting velocity, elasticity and plasticity fields agreed with experimental measurements.

This improved our understanding of Couette flows [38, 39]. The localization of the velocity field results from the stress heterogeneity, so that the circular geometry by itself can induce localisation. In planar geometry, where the stress is a priori homogeneous, localisation necessarily relies on another cause of heterogeneity, such as an external friction. Initial normal stresses can be preserved even in a steady flow, so that there are residual normal stresses which depend on initial conditions linked with the foam preparation method: the steady flow is not unique. Despite its simplicity, a Couette flow displays specific VEP features [37]. For instance, at the boundary of the localised region, the discontinuity of the velocity gradient depends on the residual normal stresses, and thus on initial conditions. For all these reasons, and because the range of experimental data is limited, Couette flows have only a limited ability to discriminate between models, or between parameter values.

\subsection{Notations and equations}

\subsubsection{Constitutive equations: solid mechanics}

We start with constitutive equations specific of a semifluid semi-solid material (see fig. 3). In order to emphasize the dominant role of elasticity, we express them here in terms of deformations and their rates, as is usual in the context of solid mechanics

$$
\begin{aligned}
\sigma_{\text {tot }} & =-p I+2 \eta_{1} \dot{\varepsilon}+2 \mu \varepsilon^{e}, \\
\dot{\varepsilon} & =\dot{\varepsilon}^{e}+\dot{\varepsilon}^{p}, \\
\dot{\varepsilon}^{p} & = \begin{cases}\frac{1}{\lambda} \frac{\left|\varepsilon^{e}\right|-\varepsilon_{Y}}{\left|\varepsilon^{e}\right|} \varepsilon^{e}, & \text { when }\left|\varepsilon^{e}\right|>\varepsilon_{Y}, \\
0, & \text { otherwise. }\end{cases}
\end{aligned}
$$

Equation (1) is a constitutive equation for the Cauchy stress $\sigma_{\text {tot }}$, with pressure, viscous and elastic terms. Here $p$ is the pressure, $I$ the identity tensor; $\dot{\varepsilon}$ is the total deformation rate tensor, $\eta_{1}$ is the viscosity of the material apparent at small deformation (in the foam, it includes the dissipation inside the soap films); $\varepsilon^{e}$ is the elastic deformation tensor, $\mu$ the shear modulus.

Equation (2) recalls that $\dot{\varepsilon}$ is shared between elastic $\dot{\varepsilon}^{e}$ and plastic $\dot{\varepsilon}^{p}$ contributions. 


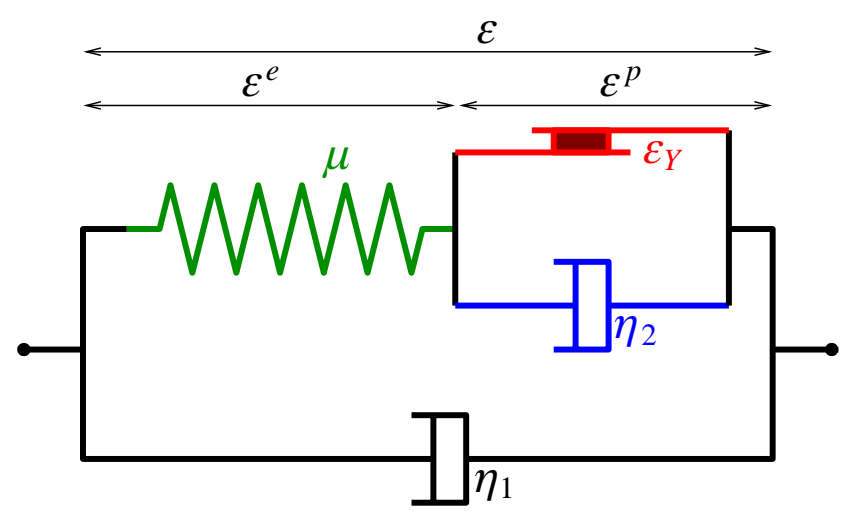

Fig. 3. Symbolic representation of the viscous, elastic, plastic (VEP) model [21]: The spring symbolises an elastic stress proportional to the elastic deformation, and stands for the elastic modulus $\mu$. The dashpots, with a viscous stress proportional to the total deformation rate, stand for viscous dissipations $\eta_{1}$, $\eta_{2}$. The solid friction element, which does not move until a sufficient force is applied, and exerts a constant resistance when it moves, stands for the plastic flow occurring when the elastic deformation is larger than the yield strain $\varepsilon_{Y}$.

Equation (3) is a plasticity equation, which specifies $\dot{\varepsilon}^{p}$ by stating that plasticity increases above the yield strain $\varepsilon_{Y}$ : under a strong shear rate, the elastic deformation $\left|\varepsilon^{e}\right|$ can become significantly higher than $\varepsilon_{Y}$. Here $\left|\varepsilon^{e}\right|$ is the norm of $\varepsilon^{e}$, which we define as $\left|\varepsilon^{e}\right|=$ $\left[\left(\varepsilon_{y x}^{e}\right)^{2}+\left(\varepsilon_{x x}^{e}-\varepsilon_{y y}^{e}\right)^{2} / 4\right]^{1 / 2}$ to facilitate the comparison with a scalar shear (fixed shear direction $x y$ with a given amplitude $\varepsilon$ ), i.e., $\varepsilon_{x x}^{e}-\varepsilon_{y y}^{e}=0$ and $\varepsilon_{y x}^{e}=\varepsilon$, so that $\left|\varepsilon^{e}\right|=\varepsilon[3]$; the externally measured scalar shear is then $\gamma=2\left|\varepsilon^{e}\right|$. Another acceptable definition would be the Euclidian norm of the deviatoric elastic strain tensor [38,39], $\left(\left[\left(\varepsilon_{x x}^{e}-\varepsilon_{y y}^{e}\right) / 2\right]^{2}+\left(\varepsilon_{x y}^{e}\right)^{2}+\left(\varepsilon_{y x}^{e}\right)^{2}+\left[\left(\varepsilon_{y y}^{e}-\varepsilon_{x x}^{e}\right) / 2\right]^{2}\right)^{1 / 2}=$ $\left[2\left(\varepsilon_{y x}^{e}\right)^{2}+\left(\varepsilon_{x x}^{e}-\varepsilon_{y y}^{e}\right)^{2} / 2\right]^{1 / 2}$, which is $\sqrt{2}$ times larger than $\left|\varepsilon^{e}\right|$. The value of $\varepsilon_{Y}$ is defined consistently with that of $\left|\varepsilon^{e}\right|$.

In soft disordered materials, plasticity is related with local rearrangements. In foams, these happen when bubbles swap neighbors and are called "T1" processes $[12$, 13,40]. They create a transient local deformation. Here $\lambda$ is the relaxation time of the material after such a local deformation [41]. We can construct the dissipation due to plasticity, which has the dimension of an effective viscosity $\eta_{2}=\lambda \mu$ : it determines the loss modulus at large amplitude. The softness and deformability of the material appears in the value of $\varepsilon_{Y}$, of order of unity, so that both the elastic and plastic behaviors are experimentally observable; its glassy (i.e. disordered) nature implies that $\lambda, \mu, \varepsilon_{Y}$ are isotropic [3].

\subsubsection{Conservation equations: fluid mechanics}

Generic conservation equations for an isothermal flow are expressed in terms of the velocity $\mathbf{v}$ and its derivatives, as is usual in the context of fluid mechanics

$$
\begin{aligned}
& \rho \dot{\mathbf{v}}=\operatorname{div} \sigma_{\text {tot }}+\mathbf{f}_{\text {ext }}, \\
& \operatorname{div} \mathbf{v}=0
\end{aligned}
$$

Equation (4) is the equation of dynamics; $\rho$ is the density, $\dot{\mathbf{v}}=\partial_{t} \mathbf{v}+\mathbf{v} \cdot \nabla \mathbf{v}$, and $\rho \dot{\mathbf{v}}$ denotes the inertia term (which we neglect below, see sect. 3.3); $\mathbf{f}_{\text {ext }}$ is the external force: in the bidimensional flow experiments studied below, the friction on the top and bottom boundaries (e.g., horizontal glass or perspex plates) is approximately $\mathbf{f}_{\text {ext }}=-k \mathbf{v}$, where $k$ is a constant. We find experimentally (see sect. 3.3) that $k$ is small enough that in the present flows the effect of $\mathbf{f}_{\text {ext }}$ is not measurable, so that we neglect it.

Equation (5) describes the incompressibility of the flow: it applies when the compressibility modulus is much higher than the shear modulus, as is the case in foams [1214], when the flow is slower than the sound velocity and when the overall pressure variation remains smaller than ambient pressure.

\subsubsection{Closing equations: linking solid and fluid mechanics}

To close the system of eqs. (1)-(3) and eqs. (4), (5) requires coupling the deformations to the velocity.

First, the total deformation rate equals the symmetrized velocity gradient $D(\mathbf{v})$

$$
\dot{\varepsilon}=D(\mathbf{v})=\frac{\nabla \mathbf{v}+\nabla \mathbf{v}^{T}}{2}
$$

Second, the time variation of the elastic deformation tensor $\varepsilon^{e}$ accounts for its advection by the flow velocity $\mathbf{v}$. The model should be objective, that is, the expression of the equations should remain the same for an observer who has a movement of translation or rotation with respect to the experiment. The advection of the elastic deformation tensor is thus described with a frame-invariant tensorial derivative [42]

$$
\dot{\varepsilon}^{e}=\frac{\mathcal{D} \varepsilon^{e}}{\mathcal{D} t}
$$

The objective derivative is $[42,21]$

$$
\frac{\mathcal{D} \varepsilon^{e}}{\mathcal{D} t}=\frac{\partial \varepsilon^{e}}{\partial t}+(\mathbf{v} \cdot \nabla) \varepsilon^{e}+\beta_{a}\left(\varepsilon^{e}, \nabla \mathbf{v}\right)
$$

where

$$
\begin{aligned}
\beta_{a}\left(\varepsilon^{e}, \nabla \mathbf{v}\right)= & \varepsilon^{e} \cdot W(\mathbf{v})-W(\mathbf{v}) \cdot \varepsilon^{e} \\
& -a\left(D(\mathbf{v}) \cdot \varepsilon^{e}+\varepsilon^{e} \cdot D(\mathbf{v})\right),
\end{aligned}
$$

Here $W(\mathbf{v})=\left(\nabla \mathbf{v}-\nabla \mathbf{v}^{T}\right) / 2$ is the antisymmetric part of the velocity gradient, and $a \in[-1,1]$ is the so-called " $a$ parameter" $[21,42]$, whose effect is discussed in sect. 3.3. 


\section{Methods}

\subsection{Experimental methods}

Experimental set-ups have been described in refs. $[11,7$, 43]. Bubble distributions are monodisperse in size and disordered in geometry (shape, number of neighbors). While bubbles pass through the field of view, no rupture is observed, and coarsening is negligible.

The wet foam is prepared by direct bubbling into the $1 \mathrm{~m}$ long channel. At its entrance in the channel, it displays normal differences in elastic deformation, $\varepsilon_{x x}^{e}-\varepsilon_{y y}^{e}$ (axis 2 in fig. 7).

For the dry foam, bubbles pass first through a chamber (in which the foam drains): this chamber enables to vary the liquid fraction over more than three decades, and homogeneizes the foam while relaxing its normal differences in elastic deformation [43].

Experimental measurements treat solid and liquid behaviors with a unique set of mutually compatible tools [7]. We follow this approach for the 750 sequential images of the wet foam $[11,7]$ and 1000 images of the dry foam $[7,43]$. We perform discrete measurements over images of bubbles which can be automatically identified using image analysis, that is, which do not touch the obstacle.

Local quantities are measured inside boxes. The size of the boxes should be small enough to capture the local spatial variations of the measured fields. It should be large enough that the number of bubbles (number of bubbles per box in an image, multiplied by the number of images) yields averages with small relative fluctuations. In our case the measurements are averaged over 6 (respectively, 2.7) bubbles in the wet (respectively, dry) foam. Dollet and Graner [11] (and Janiaud and Graner [44] in a Couette geometry) examined whether the discrete nature of the stress relaxation associated with each $\mathrm{T} 1$ process displays collective behavior effects (e.g. avalanches). Avalanche-like events at such a small scale would lead to extremely large fluctuations and/or a large asymmetry between positive and negative increments. No such signature of avalanches has been found on the scale of the boxes. The increments are almost Gaussian and the fluctuations with time of the local quantities are directly related to the finite number of bubbles inside each box. This supports the description of the flow using a continuous description based on local averages.

The entrance velocity $V$ is measured $( \pm 2 \%)$ as the average over the side of the field of view. The texture (bubble size, elongation and packing) and its variation (bubble stretching and rearrangements) enable to measure in situ the velocity gradient (not shown), the elastic deformation $( \pm 2 \%)$ and the plastic deformation rate $( \pm 7 \%)[7]$. We plot here deviatoric terms, see sect. 3.3.

\subsection{Resolution}

Equations (1)-(9) can be solved in 2D or in 3D. Their main difficulties are intrinsinc to VEP flows, independently of the details of the model. They reduce to a set of three partial differential equations with three unknowns $\left(\varepsilon^{e}, \mathbf{v}, p\right)$ and the coupled system is highly non-linear: its numerical resolution needs to be performed carefully.

Here we solve these equations in $2 \mathrm{D}$ with a finite element algorithm first used for a simple Couette circular geometry $[38,39]$, extended here to handle more complex flow domains [37]. As in 1D, the stationary solution is obtained by solving the time-dependent problem with a second-order time-splitting algorithm, already used for VE [45], generalized here to VEP; it allows to treat separatly the two main non-linearities of the equations, namely the plasticity term in eq. (3) and the stress transport term in the objective derivative (eq. (8)). Unlike in 1D [38,39], the stress transport term needs to be treated specifically by upwinding techniques; we chose a robust method, the discontinuous Galerkin scheme [46]. In addition, the nonlinearity linked to plasticity needs a much more careful discretization than for the Couette resolution $[38,39]$ to ensure a proper decreasing of the residue of the stationary problem. The spatial discretization is performed with a mixed finite-element method as in [33]. In order to get a general method suitable for any geometry, the domain is discretized with triangles.

The calculation domain is a channel, with entrance at $15 R=22.5 \mathrm{~cm}$ upstream and an exit at $30 R=45 \mathrm{~cm}$ downstream of the obstacle. The mesh, made of 1100 triangles, is locally refined near the obstacle (see fig. 4a). We start from a foam at rest and enforce the entrance velocity $V$. Unlike for most liquid flows, but in agreement with foam flow experiments [11], we use slip boundary conditions. Careful tests have been performed [37] in order to ensure that the mesh is sufficiently refined and that discretization does not affect the results presented here. Figure $4 \mathrm{~b}$ represents how the time-dependent calculations converge towards the stationary solution. In fig. 4c, the residual term decreases first rapidly, until $n=200$. It then reaches a plateau from $n=200$ to 3000 which corresponds to the advection of initial defects. It eventually reaches an asymptotical regime with a constant slope of convergence. Iterations were stopped when the residual term reached $10^{-7}$. Calculations in $2 \mathrm{D}$ run in half a day on a Intel T7300 Core 2 Duo processor ( $2 \mathrm{GHz}, 4 \mathrm{Mb}$ cache, 32 bits). The $2 \mathrm{D}$ algorithm has been validated by reproducing the $1 \mathrm{D}$ Couette calculation $[38,39]$, which runs in a few minutes.

\subsection{Choice of parameters}

The parameter with the main effect is $\varepsilon_{Y}$ : a change of $\pm 20 \%$ suffices to visibly affect the main features of the flow. In a foam, its value is expected to depend mostly on the liquid fraction and on the dimension (2D or $3 \mathrm{D})$, and possibly on the area dispersity; on the opposite, the average area and the physico-chemical properties of the foam are not expected to affect the value of $\varepsilon_{Y}$.

We have tested the effect of varying the other parameters, one by one. Like in Couette flow $[38,39]$, they barely 
(a)

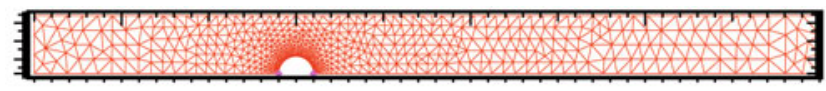

(b)

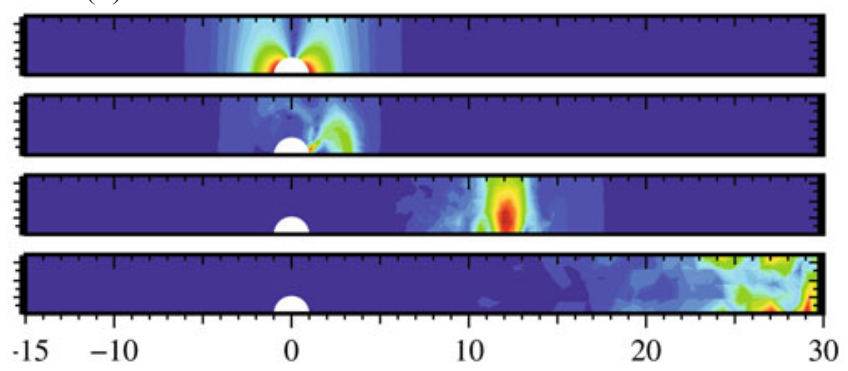

(c)

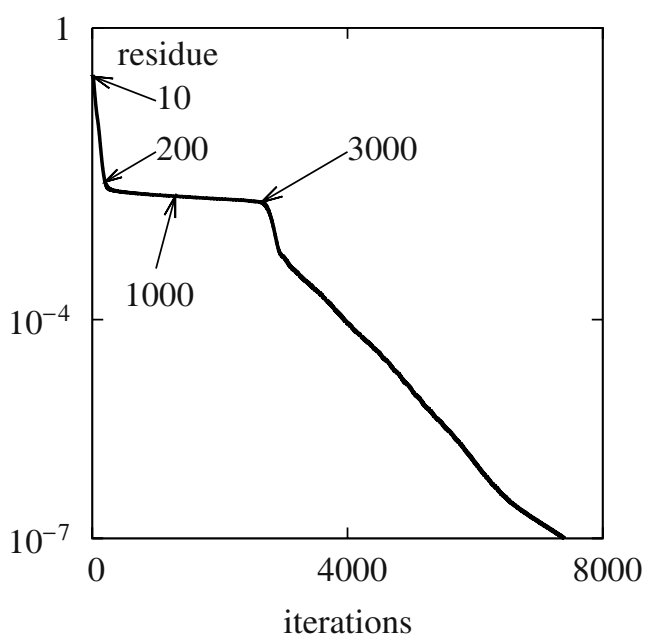

Fig. 4. Numerical convergence of calculations (see text). (a) Mesh used for the actual calculations; for convergence tests, the results have been compared with calculations performed on a finer mesh [37]. (b) The $L^{2}$ norm of the stationary residues of eqs. (1)-(5) is displayed during the calculation which led to figs. 9-12. From top to bottom: $n=10,200,1000$ and 3000 time iterations. Figures indicate $x / R$, where $R$ is the obstacle radius. (c) The same $L^{2}$ norm of the stationary residues is plotted versus time iterations.

affect the flow, even if varied over a large range, as we now discuss.

The value $k$ of the friction on the plates can be determined experimentally from the overall pressure gradient $\nabla p$ across the channel length: $k=\nabla p / V=9300 \mathrm{Pas} \mathrm{m}^{-2}$ If we take this value, we obtain a prediction indiscernable from $k=0$. Only by taking a ten times larger friction, $k=10^{5} \mathrm{Pas} \mathrm{m}^{-2}$, does the change become visible. It means that here the limit $k \rightarrow 0$ is regular. This case is similar to cylindrical Couette geometry, but different from planar Couette geometry $[38,39]$.

We choose $\eta_{1} / \eta_{2}=0.1$ according to the Couette case $[38,39]$, since it lies in the middle of a range where its exact value barely affects the flow, even up to a factor of
10. The Reynolds number $R e=\rho V R /\left(\eta_{1}+\eta_{2}\right) \approx 5 \times 10^{-3}$ is much smaller than 1 , so that we neglect the inertia term $\rho \dot{\mathbf{v}}$ in eq. (4).

We choose the co-rotational derivative [42], with $a=0$, so that $\varepsilon^{e}$ is deviatoric. In that case, the term $\beta_{a}$ in eqs. (8), (9) has a zero trace: hence if the trace of $\varepsilon^{e}$ is initially zero, it remains zero [37]. In the low-velocity regime explored here, any other choice of the objective derivative, with a parameter ranging from $a=-1$, lower-convected derivative, to $a=1$, upper-convected [42], would only change the trace of $\varepsilon^{e}$. Further investigations might determine the value of $a$ by comparison with the experimental values of this trace.

The relaxation time $\lambda$ is related to the Weissenberg number $W e=\lambda V / R$. We distinguish three velocity regimes. At high velocity, We of order 1 or higher, the material rheology can display non-linearities in addition to those already present in the model. Since foams rupture at high velocities $[43,47]$, this regime would be easier to investigate with other materials. In the low velocity range, We greater than $10^{-2}$ but smaller than 1 , the exact value of We does not affect the flow. This is the case for both experiments considered here, as well as for several foam flow experiments reported in the literature. Further decreasing We over two or three decades would lead to the ultra slow range, where the fore-aft asymmetry strongly increases. This is done in very few well-controlled experiments [43]. The limit $W e \rightarrow 0$ at constant $\varepsilon_{Y}$ is singular: it implies a divergence of the Bingham number $B i=2 \varepsilon_{Y} / W e$ [21]. In fact, the ultra slow regime is not quasi-static $[15,18,48]$. and does not exactly match quasi-static simulations [18].

\subsection{Representations}

Results are plotted either as maps or graphs.

Maps enable a global quantitative comparison between calculations and experiments: spatial distribution, magnitude, orientation of different quantities. Velocities are displayed as arrows. For clarity, they are presented in the average reference frame of the foam, as if a movable obstacle was displaced towards the left within a fixed foam, i.e. $\left(v_{x}-V\right) / V$. Traceless tensors are represented by circles. Their two eigenvalues have the same absolute value. We indicate the direction of the positive one by a thin line. The direction of the negative one, not represented, is perpendicular. The top half, in red, is the present calculation using a continuous model, performed in the half-plane assuming top-bottom symmetry. Thin lines are isovalues of the stream function. The bottom half, in blue, are experimental data obtained as averages over bubbles. Thin lines are trajectories deduced from the experimental velocity field, plotted with approximately the same density as in calculations.

Graphs of components along selected axes enable detailed quantitative comparisons between calculations and experiments: position and amplitude of extrema, shape of curves, concavity, inflexion points, precision of the agreement. Axes are chosen to coincide with the measurements of ref. [11]: axis 1 is the symmetry axis, passing through 


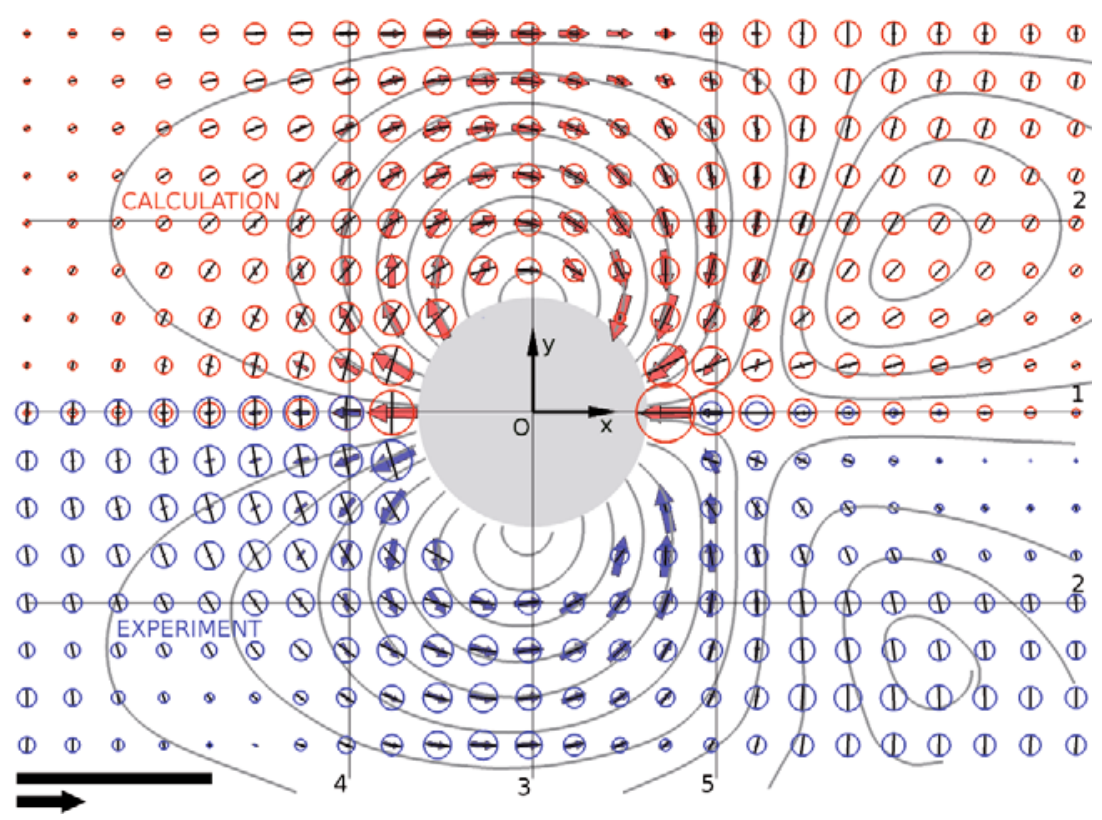

Fig. 5. (Colour on-line) Velocity and elastic deformation of wet foam, represented as explained in sect. 3.4. Thick colored arrows: velocity field. Circles: elastic deformation tensors; the positive (respectively, negative) eigenvalue corresponding to elongation (respectively, compression) is represented by a line (respectively, not represented). Thin lines: stream lines. Top half (red): present calculation using the continuous model (same data as fig. 2 ): $\varepsilon_{Y}=0.1, \lambda=0.2 \mathrm{~s}, \eta_{1} / \eta_{2}=0.1, k=0$. Bottom half (blue): experimental data obtained as averages over bubbles shown in fig. 1a. Scale: bar: 1 (dimensionless) for the elastic eigenvalues (circle diameter); black arrow: entrance velocity $V$.

the obstacle center, parallel to the flow, $y=0$; axis 2 is parallel to the flow, close to the obstacle $y= \pm 2.5 \mathrm{~cm}$; axis 3 is perpendicular to the flow, passing through the obstacle center, $x=0$; axis 4 is perpendicular to the flow, upstream of the obstacle, $x=-2.4 \mathrm{~cm}$; axis 5 is perpendicular to the flow, downstream of the obstacle, $x=2.4 \mathrm{~cm}$. We do not plot components which vanish due to symmetry, such as $v_{y}$ or $\varepsilon_{x y}^{e}$ along the symmetry axis. Red lines are predictions, blue symbols are experimental tests.

\section{Results}

\subsection{Wet foam flow}

First, as a preliminary characterization, we study the flow of a wet foam ( $7 \%$ liquid fraction, fig. 1a). We calculate the measurable fields: velocity $\mathbf{v}$, elastic deformation $\varepsilon^{e}$, plastic deformation rate $\dot{\varepsilon}^{p}$. We use the parameters obtained in refs. [38,39] and rescale them to the geometry of the present set-up. We investigate separately the effect of each parameter (see sect. 3.3). We check that, with a yield strain $\varepsilon_{Y}=0.1 \pm 0.02$, the calculations agree well simultaneously with all available experimental data (fig. 2 and figs. 5-8). Such value of $\varepsilon_{Y}$ is the expected order of magnitude for a foam with this liquid fraction [7].
Other parameters have less effect. We use $\lambda=0.2 \pm$ $0.1 \mathrm{~s}$ [11] and $\eta_{1} / \eta_{2}=0.1$. We take $k=0$ without significantly affecting the results. With a shear modulus estimated [11] around $\mu=13 \pm 1 \mathrm{~N} \mathrm{~m}^{-2}$, this translates into actual values: $\sigma_{Y}=2 \mu \varepsilon_{Y}=2.6 \mathrm{~N} \mathrm{~m}^{-2}, \eta_{2}=\lambda \mu=2.6 \mathrm{Pas}$ and $\eta_{1}=0.1 \eta_{2}=0.26 \mathrm{Pas}$.

In such a VEP flow, elastic deformations are present in the wake of the obstacle at arbitrarily low velocity. Plasticity prevents the increase of extensional deformation, breaking the up-downstream (fore-aft) symmetry (fig. 2). A velocity overshoot, the so-called "negative wake", is clearly visible behind the obstacle (fig. 2). This characteristic feature of VEP flows is barely affected even if we vary $V$ across the low-velocity regime, confirming experimental observations [11]. For instance, dividing $V$ by 20 barely changes the overshoot (fig. 2).

This strongly contrasts with VP flows, which are always fore-aft symmetric [33]. VE flows represent a mixed situation, where the negative wake has already been evidenced, both experimentally [49] and numerically [50,51]. In fact, it occurs for low extensional viscosity fluids and models (e.g., FENE-CR [51] but not Oldroyd-B [34]), at elongational rates large enough in comparison with the inverse relaxation time, so that the elastic deformation does not vanish. However, at the low velocity investigated here, the VE flow is completely fore-aft symmetric (fig. 2), and even indiscernible from viscous flows, whatever the viscosity. To interpret this set of observations, it seems that the overshoot appears when the elastic de- 

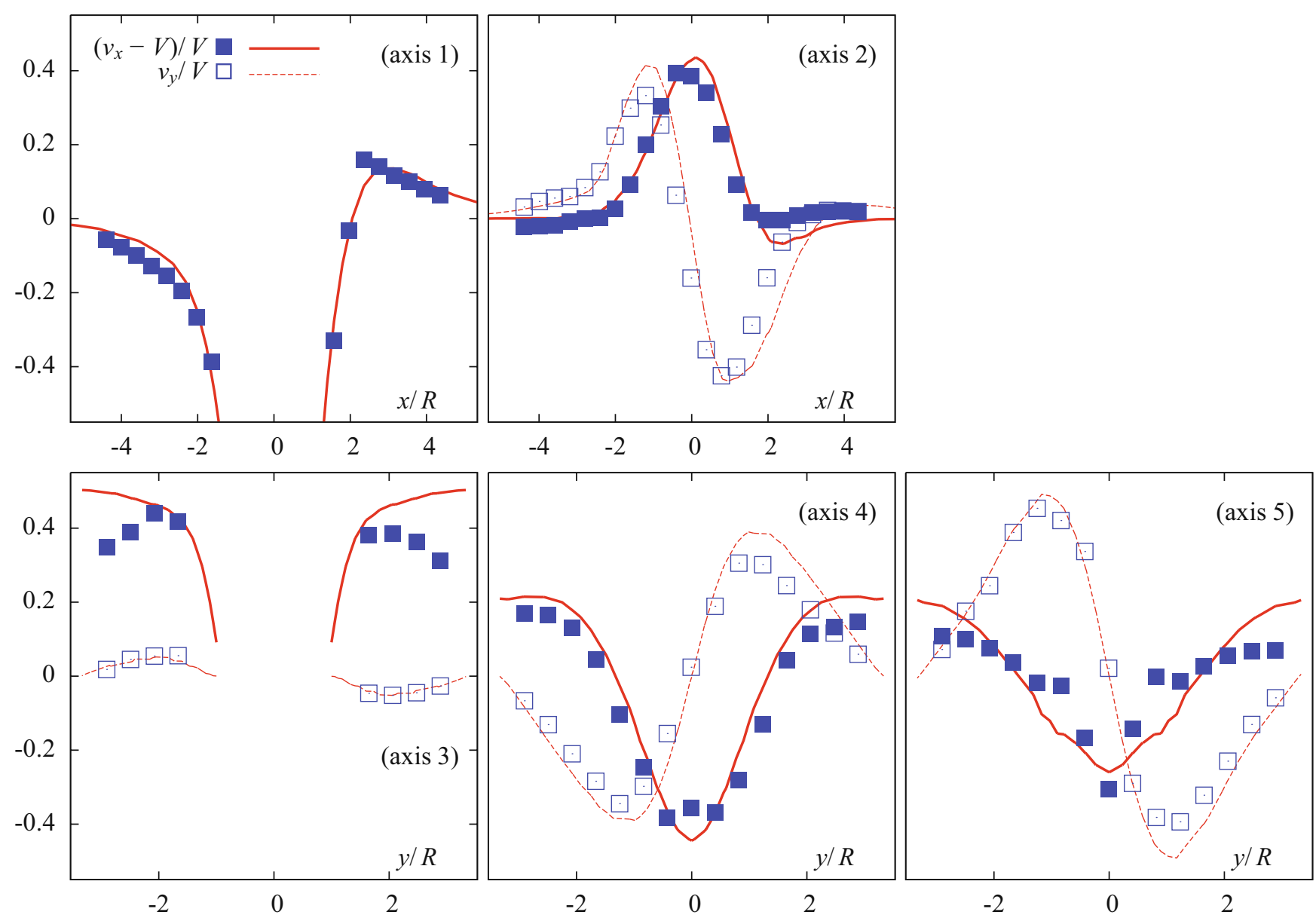

Fig. 6. (Colour on-line) Velocity of wet foam (same data as fig. 5): plots along axes 1-5 (shown in fig. 5). Red lines are predictions, blue symbols are experimental data. $\left(v_{x}-V\right) / V$ : solid thick lines and closed squares; $v_{y} / V$ : dashed thin lines and open squares.

formation ceases to follow passively the total deformation rate. This can occur if there is a mechanism which saturates the value of elastic deformation. In VE materials, such mechanism exists in some models [50,51], but only at high velocity; at low velocity, it disappears in all models. Conversely, in VEP materials the overshoot amplitude directly depends on $\varepsilon_{Y}$ : such materials seem to exhibit an elastic response at any velocity, even arbitrarily low.

To summarize this first test, by adjusting only one parameter, whose value has the expected order of magnitude, we can adjust both qualitative and quantitative features of all available data. We reproduce the observed negative wake and evidence the specificity of VEP flows.

\subsection{Dry foam flow}

Second, we turn to prediction. Since the parameter which has the most significant effect on the flow is the yield strain [11] (sect. 3.3), we choose to predict the flow for a twice larger value, $\varepsilon_{Y}=0.2$.
This prediction is plotted in figs. 9-12. The overshoot on the symmetry axis is larger and closer to the obstacle than for $\varepsilon_{Y}=0.1$ (fig. 13): this reflects a larger effect of the elastic deformation.

To check this prediction, we then perform a dry foam experiment, since decreasing the liquid fraction of a foam is expected to affect especially the yield strain. With $1.2 \%$ liquid fraction, (fig. 1b), we observe that $\varepsilon_{\max }^{e}$, the maximum value of $\varepsilon^{e}$ measured on the experiment, is twice as much that of the wet foam. The effect of elasticity is even stronger and the agreement with our prediction even better, without adjusting any parameter. Measurements confirm the predicted spatial distribution, magnitude, direction, anisotropy of fields.

The bubble velocity (figs. 9-10) passes the three most stringent tests. First, the position and magnitude of overshoot on the symmetry axis (fig. 10). Second, the graph along the axis 5 (fig. 10). And third, the exact position of the arrest points, defined in the reference frame of the foam, as points where $v_{x}-V=0$ : close to axis 5 , on $y=0$ and $\pm 5 \mathrm{~cm}$, see fig. 9 . Other axes confirm the agreement. 

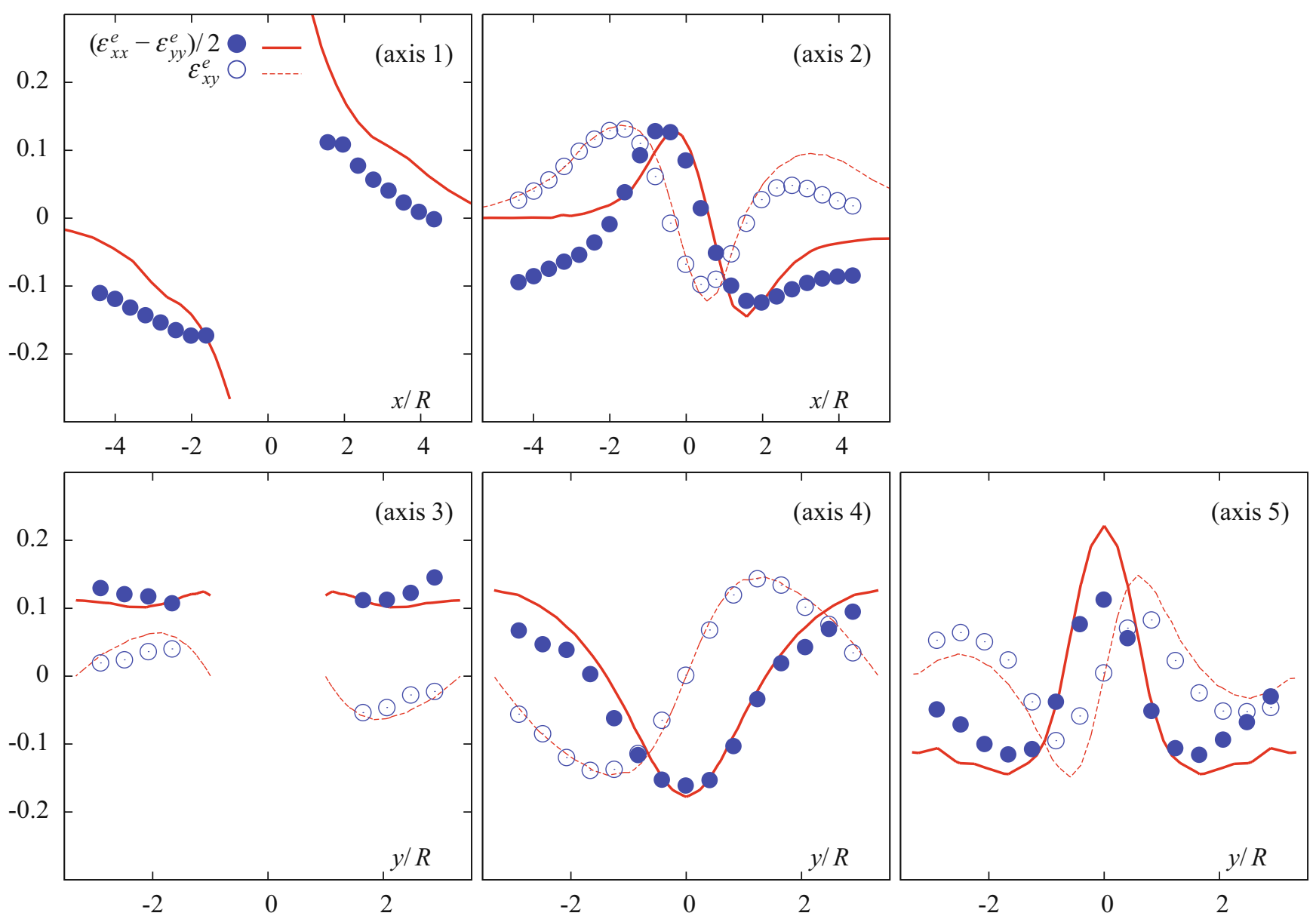

Fig. 7. (Colour on-line) Elastic deformation of wet foam (same data as fig. 5): plots along axes 1-5 (shown in fig. 5). Red lines are calculations, blue symbols are experimental data: $\left(\varepsilon_{x x}^{e}-\varepsilon_{y y}^{e}\right) / 2$ : solid thick lines and closed circles; $\varepsilon_{x y}^{e}$ : dashed thin lines and open circles.

The bubble elastic deformation too agrees remarkably well in spatial distribution, amplitude and direction (figs. 9-11). Interestingly, its orientation does not directly correlate with that of streamlines, or equivalently of the velocity vectors (fig. 9). In addition, the elastic deformation field extends more than the velocity gradient field (fig. 9), which itselfs extends more than the plastic deformation rate field (fig. 12). The three fields thus have a different spatial extension [7]. Specifically, the elastic deformation is not determined solely by the velocity gradient (fig. 14). This strongly contrasts with the scalar, stationary case where the elastic deformation can be eliminated and the rheology expressed as a function of velocity gradient only (as, e.g., with the Herschel-Bulkley model [27]). Note that residual normal stresses are visible beyond the obstacle. Unlike in the Couette case $[38,39]$, here they are reproducible and their origin is understood: they are a direct effect of the obstacle, and do not depend on the foam preparation method.

The plastic deformation rate is calculated as the total deformation rate minus the elastic deformation rate (eq. (2)). Its spatial distribution and directions agree with that of the experimental measurements, which represent the time-averaged orientation, frequency and anisotropy of the bubble rearrangements (fig. 12).

\section{Discussion and conclusion}

To summarize, a continuous description of viscous, elastic, plastic material with physically meaningful parameters can reproduce and even successfully predict a tensorial, spatially developed flow of a disordered rearranging structure.

We analyse and interpret the effect of each parameter separately. We emphasize the dominant role of elasticity and thus identify the yield strain as the most important parameter. The flow does not reduce to VE or VP separately, so that we emphasize the specific complexity of VEP materials.

Our method opens the way to computing two- or threedimensional flows under any type or amplitude of deformation. It applies to those depending on one space variable: for instance a flow through a channel $[28,29]$, or 


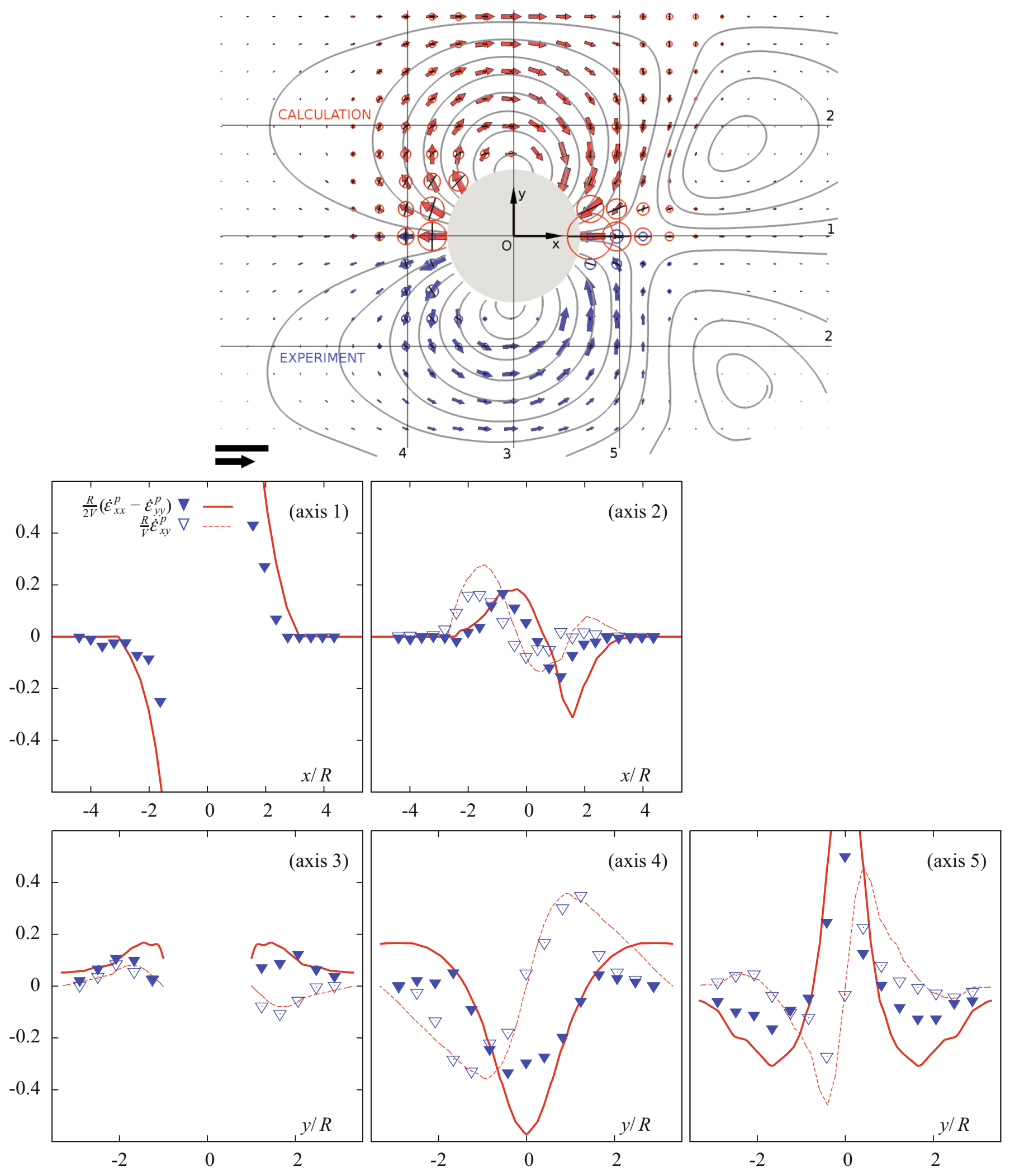

Fig. 8. Plastic deformation rate and velocity of wet foam (same data as fig. 5). Top: plastic deformation rate field (same symbols as fig. 5). Scale: bar: $1 \mathrm{~s}^{-1}$ for the plastic deformation rate eigenvalues (circle diameter); black arrow: entrance velocity $V$. Axes 1 to 5 are indicated. Bottom: plots along axes 1-5. Red lines are calculations, blue symbols are experimental data: $\left(\dot{\varepsilon}_{x x}^{p}-\dot{\varepsilon}_{y y}^{p}\right) R / 2 V$ : solid thick lines and closed triangles; $\dot{\varepsilon}_{x y}^{p} R / V$ : dashed thin lines and open triangles. 


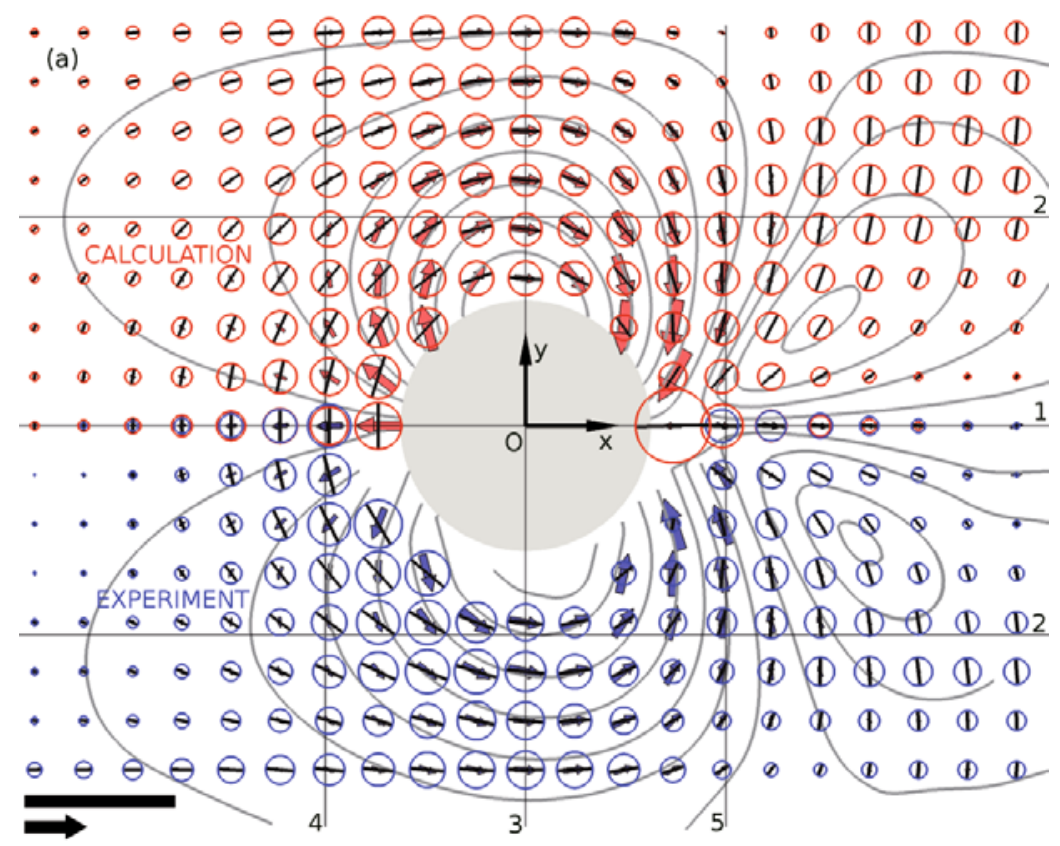

Fig. 9. Test of dry foam flow prediction $\left(\varepsilon_{Y}=0.2\right)$ : velocity and elastic deformation map (same symbols as fig. 5). Top half (red): present calculation using a continuous model. Bottom half (blue): experimental data obtained as averages over bubbles shown in fig. 1b. Scale: bar: 1 (dimensionless) for the elastic eigenvalues (circle diameter); black arrow: entrance velocity $V$.

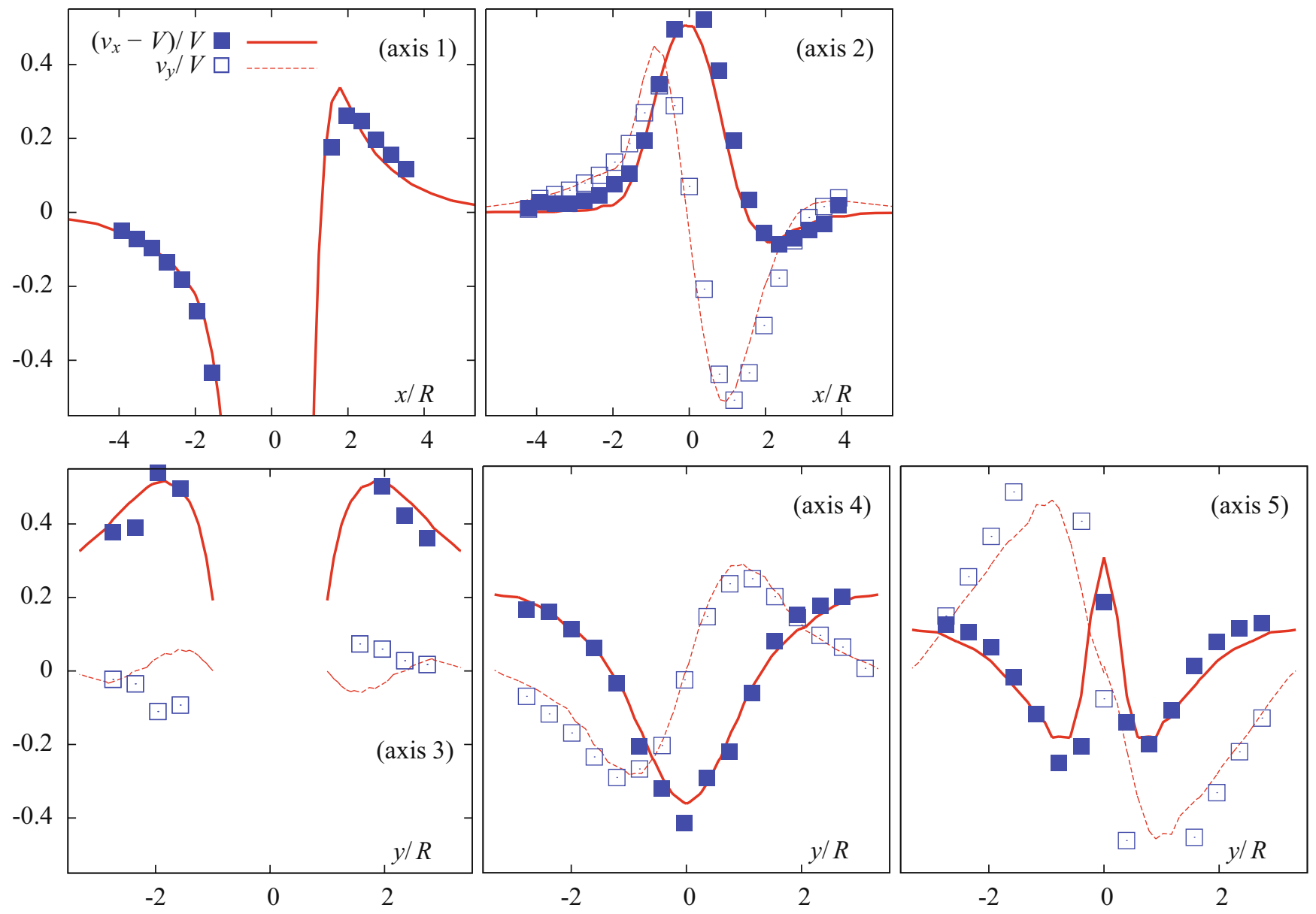

Fig. 10. Velocity of dry foam $\left(\varepsilon_{Y}=0.2\right)$ : plots along axes 1-5 (shown in fig. 9). Same data as fig. 9, same symbols as fig. 6 . 

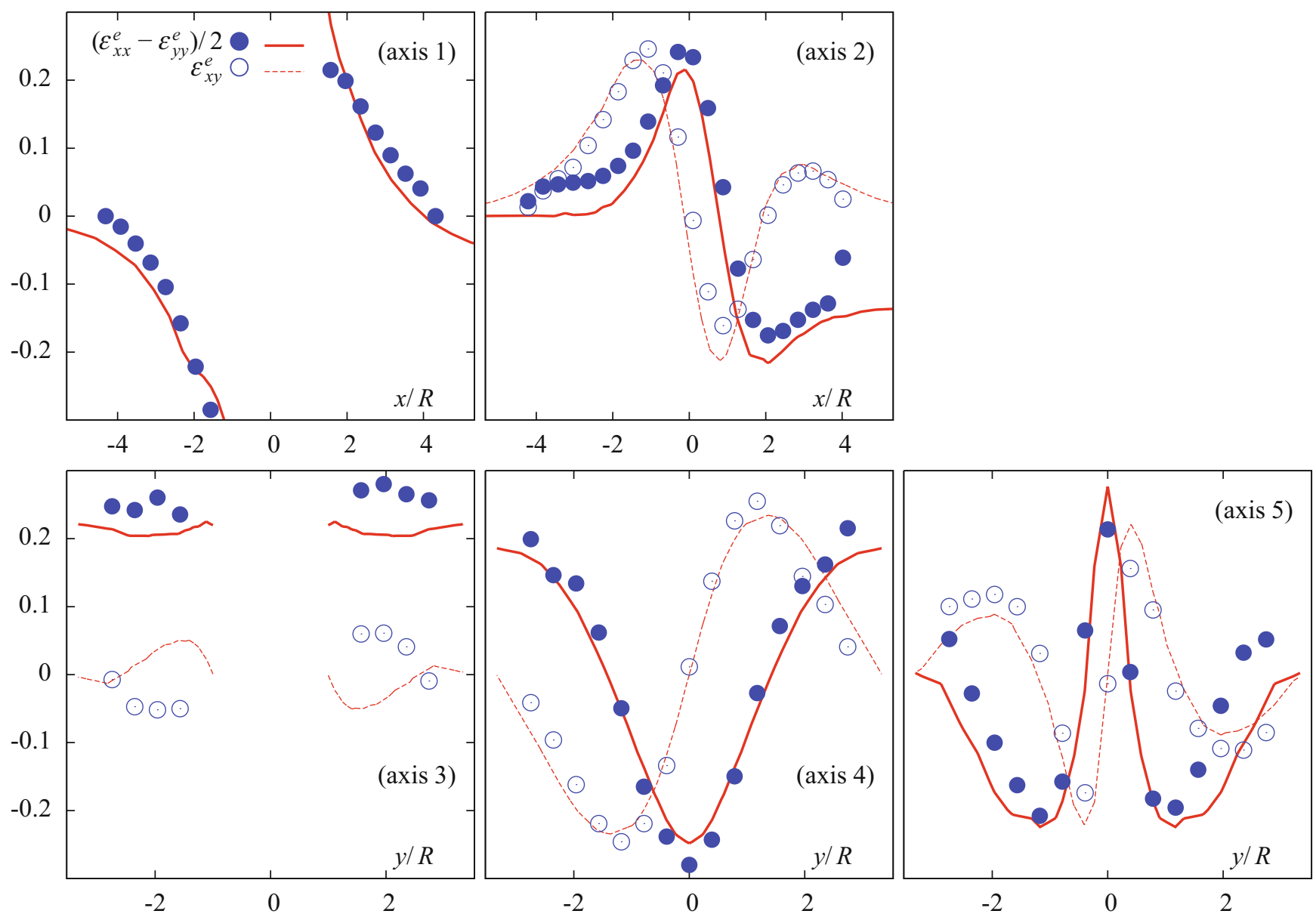

Fig. 11. Elastic deformation of dry foam $\left(\varepsilon_{Y}=0.2\right)$. Same data as fig. 9, same symbols as fig. 7 .

during simultaneous squeezing and shearing [3]. It also applies to those depending on two space variables: for instance a flow through a hole in $2 \mathrm{D}[7,52]$, or in $3 \mathrm{D}$ with axisymmetry. At the expense of longer calculations, it can even apply to flows which depend on three space variables, for instance through a twisted or branching pipe.

The model has been kept as simple as possible. On the one hand, if we suppress one or another of its ingredients, our algorithm still can solve it, and the resulting solution lacks some of the experimental features. On the other hand, it can be progressively enriched by incorporating additional non-linearities, for instance related with the physico-chemistry of foams. When the velocity or the rigidity of the surfactant layer increases, shear thinning effects [16] could be introduced by an extended version of the VEP model [53] based on an Herschel-Bulkley viscoplasticity [27] instead of a Bingham one, as observed in $[17,54]$. The friction on walls too scales non-linearly with high velocity [16]. Nonlinear elasticity at large deformation, although seldom reached in foams [7], can too be taken into account [19, 14]. At low velocity, plasticity seems to appear progres- sively: some bubbles begin to rearrange below the yield strain [7].

More generally, the model can be adapted specifically to any given VEP material of known properties. The value of the parameters of eqs. (1)-(9) depends on the microstructure, its disorder and its physico-chemical properties. This is where the present approach can be enriched by statistical models based on the micro-structure $[4,28,29$, $9,10,14,48,55]$.

We thank Y. Jiang for discussions at the beginning of this work, and Y. Bellaïche, I. Bonnet, L. Courbin, S. Cox, C. Gay, J.-M. Ghidaglia, P. Marmottant, F. Molino, C. Quilliet, H. Tabuteau for useful comments. The CNRS has funded trips within the Groupe de Recherche (GDR) "Mousses".

Open Access This article is distributed under the terms of the Creative Commons Attribution Noncommercial License which permits any noncommercial use, distribution, and reproduction in any medium, provided the original author(s) and source are credited. 

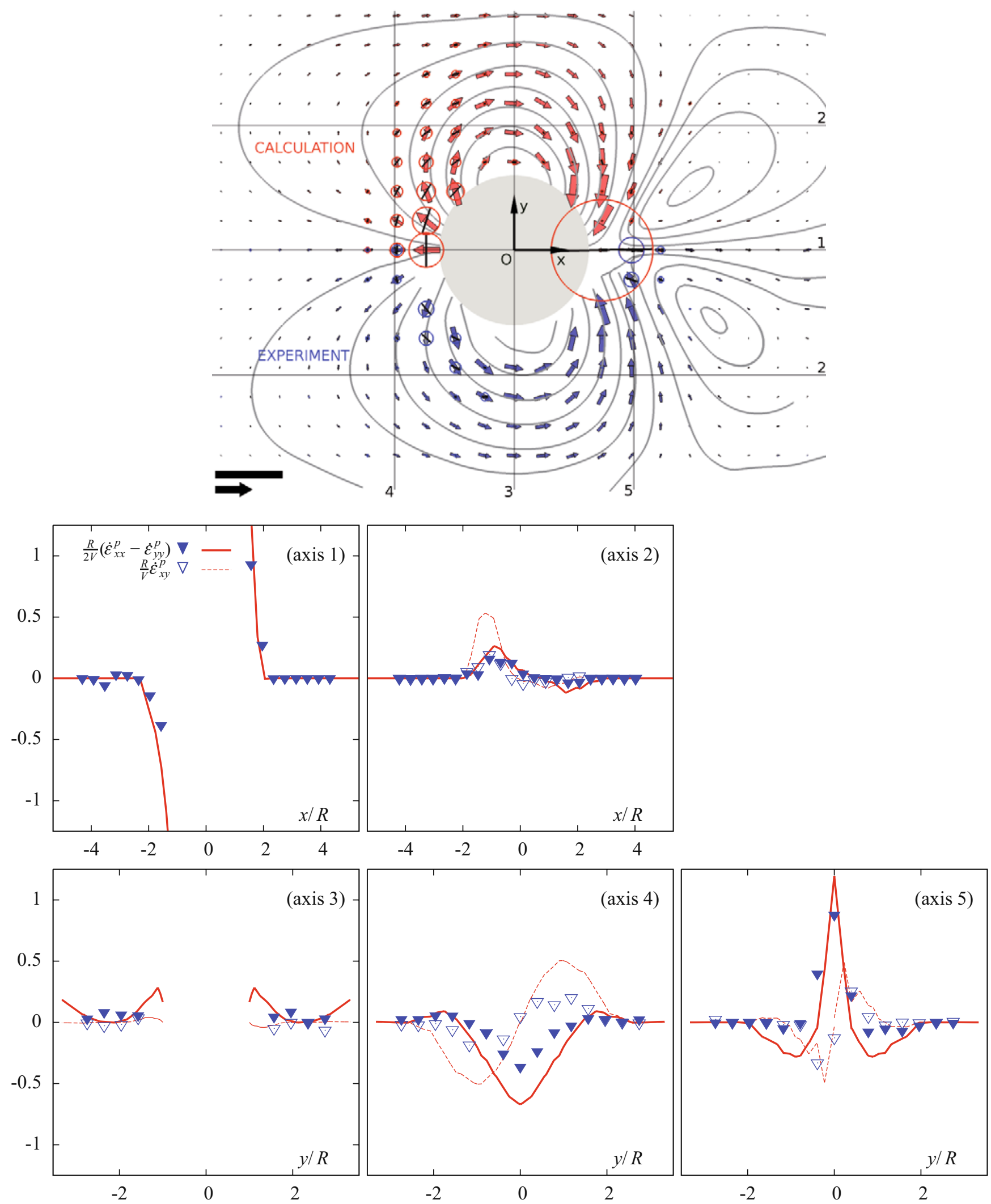

Fig. 12. Plastic deformation rate of dry foam $\left(\varepsilon_{Y}=0.2\right.$, same as in figs. 1 b and 9$)$. Top: plastic deformation rate field, same symbols as fig. 9. Scale: bar: $1 \mathrm{~s}^{-1}$ for the plastic deformation rate eigenvalues (circle diameter); black arrow: entrance velocity $V$. Bottom: plots along axes 1-5. Same symbols as fig. 8 . 


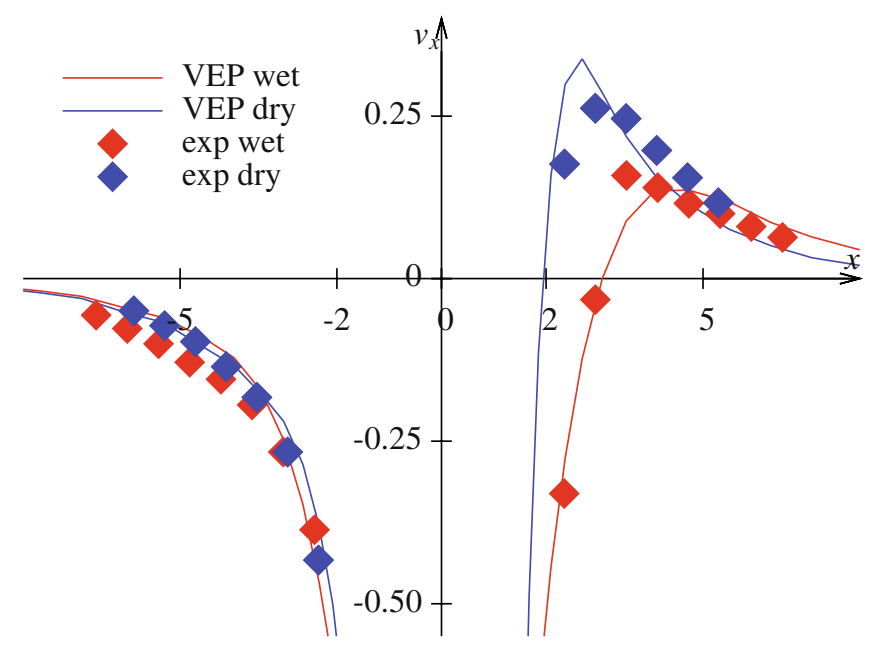

Fig. 13. Comparison between the overshoots in dry and wet foams (experiments and models): plot of the component $v_{x}$ of velocity along axis 1 .

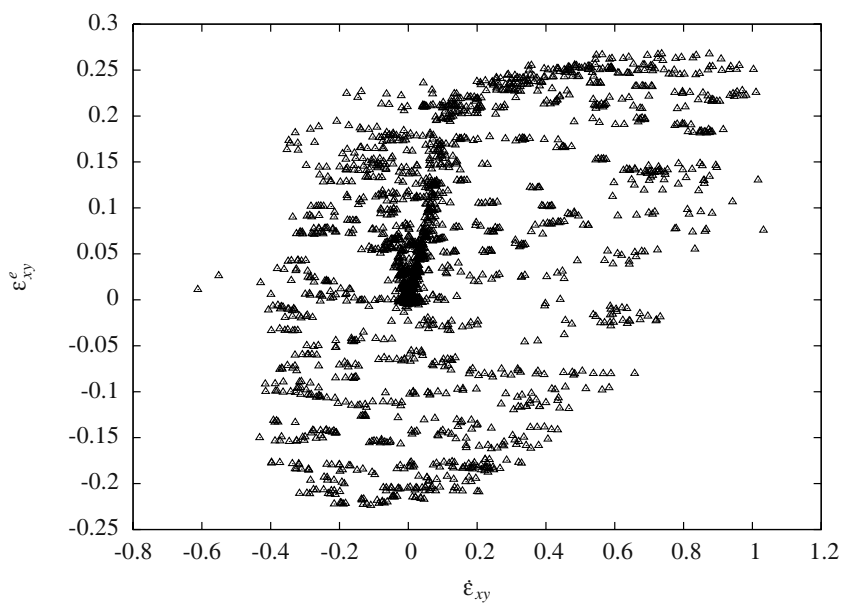

Fig. 14. Absence of a one-to-one correlation between the elastic deformation $\varepsilon^{e}$ and the velocity gradient $\dot{\varepsilon}=D(v)$ in the VEP model. Data correspond to calculations for the dry foam. For each point of the mesh used for the actual computation (fig. 4a), we plot the shear component $\varepsilon_{x y}^{e}$ of the elastic deformation vs. the shear component $\dot{\varepsilon}_{x y}$ of the velocity gradient. The points do not gather on a master curve as would be the case with, e.g., the Herschel-Bulkley model [27].

\section{References}

1. P. Coussot, Rheometry of Pastes, Suspensions and Granular Materials (Wiley, 2005).

2. D. Csontos, Nature 464, 175 (2010).

3. G. Ovarlez, Q. Barral, P. Coussot, Nature Mater. 9, 115 (2010).

4. P. Sollich, Phys. Rev. E 58, 738 (1998).

5. P. Sollich, F. Lequeux, P. Hébraud, M.E. Cates, Phys. Rev. Lett. 78, 2020 (1997).

6. M.E. Cates, P. Sollich, J. Rheol. 48, 193 (2004).

7. P. Marmottant, C. Raufaste, F. Graner, Eur. Phys. J. E 25, 371 (2008).
8. P. Marmottant, A. Mgharbel, J. Käfer, B. Audren, J.P. Rieu, J.C. Vial, B. van der Sanden, A.F.M. Marée, F. Graner, H. Delanoë-Ayari, Proc. Natl. Acad. Sci. U.S.A. 106, 17271 (2009).

9. T. Okuzono, K. Kawasaki, Phys. Rev. E 51, 1246 (1995).

10. E. Pratt, M. Dennin, Phys. Rev. E 67, 051402 (2003).

11. B. Dollet, F. Graner, J. Fluid Mech. 585, 181 (2007).

12. D. Weaire, S. Hutzler, The Physics of Foams (Oxford University Press, Oxford, 2000).

13. I. Cantat, S. Cohen-Addad, F. Elias, F. Graner, R. Höhler, O. Pitois, F. Rouyer, A. Saint-Jalmes, Les Mousses - Structure et Dynamique (Collection Echelles, Belin, 2010).

14. R. Höhler, S. Cohen-Addad, J. Phys.: Condens. Matter 17, R1041 (2005).

15. P. Marmottant, F. Graner, Eur. Phys. J. E 23, 337 (2007).

16. N.D. Denkov, S. Tcholakova, K. Golemanov, K.P. Ananthpadmanabhan, A. Lips, Soft Matter 5, 3389 (2009).

17. G. Katgert, M.E. Möbius, M. van Hecke, Phys. Rev. Lett. 101, 058301 (2008).

18. E. Janiaud, D. Weaire, S. Hutzler, Phys. Rev. Lett. 97, 038302 (2006).

19. S. Benito, C.H. Bruneau, T. Colin, C. Gay, F. Molino, Eur. Phys. J. E 25, 225 (2008).

20. L. Preziosi, D. Ambrosi, C. Verdier, J. Theor. Biol. 262, 35 (2010).

21. P. Saramito, J. Non-Newtonian Fluid Mech. 145, 1 (2007).

22. J.M. Brader, M.E. Cates, M. Fuchs, Phys. Rev. Lett. 101, 138301 (2008).

23. J.M. Brader, T. Voigtmann, M. Fuchs, R.G. Larson, M.E. Cates, Proc. Natl. Acad. Sci. U.S.A. 106, 15186 (2009).

24. P.D. Olmsted, O. Radulescu, C.Y.D. Lu, J. Rheol. 44, 257 (2000).

25. L. Bocquet, A. Colin, A. Ajdari, Phys. Rev. Lett. 103, 036001 (2009).

26. E.C. Bingham, Fluidity and Plasticity (McGraw-Hill, 1922).

27. W.H. Herschel, T. Bulkley, Am. Soc. Test Proc. 26, 621 (1926).

28. J. Goyon, A. Colin, G. Ovarlez, A. Ajdari, L. Bocquet, Nature 454, 84 (2008).

29. J. Goyon, A. Colin, L. Bocquet, Soft Matter 6, 2668 (2010).

30. T. Gibaud, C. Barentin, S. Manneville, Phys. Rev. Lett. 101, 258302 (2008).

31. G.G. Stokes, Cambr. Philos. Soc. Trans. 9, 8 (1851).

32. H. Tabuteau, P. Coussot, J. de Bruyn, J. Rheol. 51, 125137 (2007).

33. N. Roquet, P. Saramito, Comput. Appl. Meth. Mech. Eng. 192, 3317 (2003).

34. J.G. Oldroyd, Proc. R. Soc. London, Ser. A 200, 523 (1950).

35. G. Debrégeas, H. Tabuteau, J.M. di Meglio, Phys. Rev. Lett. 87, 178305 (2001).

36. Y. Wang, K. Krishan, M. Dennin, Phys. Rev. E 73, 031401 (2006).

37. I. Cheddadi, Ph.D. thesis, Université de Grenoble, http://tel.archives-ouvertes.fr/tel-00497436/en/ (2010).

38. I. Cheddadi, P. Saramito, C. Raufaste, P. Marmottant, F. Graner, Eur. Phys. J. E 27, 123 (2008).

39. I. Cheddadi, P. Saramito, C. Raufaste, P. Marmottant, F. Graner, Eur. Phys. J. E 28, 479 (2009).

40. D. Weaire, N. Rivier, Contemp. Phys. 25, 59 (1984). 
41. M. Durand, H.A. Stone, Phys. Rev. Lett. 97, 226101 (2006).

42. R.J. Gordon, W.R. Schowalter, J. Rheol. 16, 79 (1972).

43. C. Raufaste, Ph.D. thesis, Université de Grenoble, http://tel.archives-ouvertes.fr/tel-00193248/en/ (2007).

44. E. Janiaud, F. Graner, J. Fluid Mech. 532, 243 (2005).

45. P. Saramito, J. Non Newtonian Fluid Mech. 60, 199 (1995).

46. P. Lesaint, P.A. Raviart, A finite element method for solving the neutron transport equation, in Mathematical Aspects of Finite Elements in Partial Differential Equations, edited by C. Boor (Academic Press, New York, 1974) pp. 89-123.
47. B. Dollet, I. Cantat, J. Fluid Mech. 652, 529 (2010).

48. M.E. Möbius, G. Katgert, M. van Hecke, EPL 90, 44003 (2010).

49. M.T. Arigo, G.H. McKinley, Rheol. Acta 37, 307 (1998).

50. H.S. Dou, N. Phan-Thien, Rheol. Acta 42, 383 (2003).

51. A. Afonso, M. Alves, F. Pinho, P. Oliveira, Rheol. Acta 47, 325 (2008).

52. B. Dollet, J. Rheol. 54, 741 (2010).

53. P. Saramito, J. Non Newtonian Fluid Mech. 158, 154 (2009).

54. G. Katgert, A. Latka, M.E. Möbius, M. van Hecke, Phys. Rev. E 79, 066318 (2009).

55. G. Katgert, B.P. Tighe, M.E. Möbius, M. van Hecke, EPL 90, $54002(2010)$. 